who mentioned carbonate of guaiacol and carbonate of creosote as " useful alternatives" for creosote and guaiacol. I believe, rather, that in every case instead of the toxic and corrosive creosote and guaiacol the non-toxic and noncorrosive carbonate of creosote and carbonate of guaiacol should be given. Professor Dujardin-Beaumetz, in his "Dictionnaire de Therapeutique," 1895, says : "Creosote is recognised as the most efficient agent we possess for the treatments of tuberculosis, but being caustic it can only be given in small doses. This objection does not hold for 'creosotal' and carbonate of guaiacol. 'The creosote in it is in such combination that doses formerly believed impossible can be administered, hence their administration constitutes a most important advance in the treatment of tuberculosis. As creosote is not better borne in any other form 'creosotal' and carbonate of guaiacol are to be preferred in all repects in the treatment of phthisical cases."

Dresden.

\section{GANGRENE COMPLICATING PUERPERAL MANIA.}

BY E. R. ROUSE, M.R.C.S. ExG., L.R.C.P. LoNd, ASSISTANT MLEDIOAL OFFIOER, LONDON COUNTY ASTLUM, COLNEY HATCH.

ALTHovgh puerperal mania is one of the commonest forms of insanity admitted to our asylums gangrene as a complication is almost unheard of. Taking the records of our cases for the last ten years there is not a case quoted, and this alone, I think, is fairly conclusive evidence considering the great number of female admissions we have suffering from puerperal mania every year. The two following cases, coming as they did within a week of each other coupled with their rarity and ultimate recovery, are, I think, worthy of record.

CASE 1.-A woman aged thirty-one years was admitted on Jan. 10th, 1896, in a condition of acute mania the result of her confinement two weeks previously. It was her first child and the labour had been (as far as could be gathered) quite natural. On admission her mental symptoms were of a most severe nature; she was extremely restless and noisy, constantly shouting in an incoherent manner, and having very little sleep without the aid of narcotics. There was no hereditary history to be made out and she appeared to have been free from illness. Her physical state presented nothing abnormal and she was well nourished. She remained in a state of acute excitement for about a week, when her symptoms abated considerably. She, however, now complained of great pain in her feet and fingers, which on examination were quite white, cold, and anesthetic. Hot bottles were applied and the limbs were elevated and wrapped in several layers of cotton-wool. On the following morning the toes of both feet and the fingers of one hand and the right ear were, it was evident, in a condition of gangrene, and the case simulated very closely one of Raynaud's disease. Excepting for a slight elevation of temperature $\left(99^{\circ} \mathbf{F}\right.$.) she seemed fairly well in herself; there was no albumin or sugar in her urine and nothing physically to be made out. On questioning the patient she stated, however, that she had a slight vaginal discharge which smelt very offensive and which had only just appeared, the lochia having ceased before admission (which, however, I should think was doubtful). The gangrene in the feet slowly but surely advanced, clearing up, however, entirely in her fingers and right ear. Her temperature, particularly in the evening, now was elevated, reaching sometimes $102^{\circ}$, and she was becoming somewhat thinner, being unable to take much nourishment and suffering severe pain. A good nourishing diet was ordered, opium was given to relieve the pain, and the vagina was constantly syringed with permanganate of potash and warm water. In spite of all treatment the case progressed unfavourably and she had now many signs of septic poisoning. Her condition becoming serious amputation of both legs was proposed to the friends, who consented to the operation being done. With the assistance of my colleague, Mr. Robinson, we removed both feet, a modified circular flap method being done in the lower third of the right leg and Syme's amputation in the left. She bore the anæsthetic well, and on the evening of the operation, though complaining of some pain, appeared fairly comfortable, her temperature being $99^{\circ}$. She progressed favourably for about three days, when the temperature reached $101^{\circ}$, and it was considered advisable to re-dress the stump. This was done, and unfortunately in both stumps a localised patch of recurrent gangrene about the size of a shilling bad presented itself. The wound was thoroughly cleansed with perchloride of mercury and dressed with iodoform powder, double cyanide gauze, and wool. After this the case progressed favourably, the patches slowly separating and the wound healing by granulation at these spots. On March 16th she was measured for false feet and on April. 17th, about three months after ber admission, she was discharged recovered. Since her discharge I learn that mentally she is quite well and with slight assistance is able to get about her daily duties.

CASE 2. - This case, although simulating the first in many points, was more serious from the onset, the patient's mental state being extremely bad, and this, coupled with a more feeble constitution, left her in a very exhausted condition. The patient was aged thirty-seven years and the mother of five children, the last of whom was born three months previously to her admission to the asylum on Jan. 29th, 1896 She was in an acufely maniacal state (this was her second attaok); there was no hereditary history obtainable, and her physical condition, excepting a slight bronchial catarrb. was normal. She had had one miscarriage. Her condition remained, as regards her mental state, very unsatisfactory, and on Feb. 12th, about a fortnight after admission, her right foot and the anterior surface of her right leg appeared quits cold and of a dark ashen colour; at the same time the patient complained of severe pain. Peculiarly enough, as in the first case, ber mental symptoms now greatly improved. The limb was treated in exactly the same manner as in Case 1 and a nourishing diet ordered with a mixture containing carbonate of ammonia and cinchona. In spite of ail treatment the gangrene advanced, and much more rapidly than in the first case, and as it was quite evident she would be quite unable to cope with any long suppuration, owing to her exhausted condition, amputation was advised as soon as a line of demarcation appeared. The disease extended to just below the knee, running higher, however, on the outside than the inside of the limb. The leg was removed on Feb. 26th in the lower third of the thigh. The patient took the anæsthetic fairly well, but was extremely exhausted on the evening of the operation, her temperature advancing to $100 \cdot 4^{\circ} \mathrm{F}$. She had an injection of morphia that night and slept fairly well. For some few days the patient remained in a most precarions state, having a very high temperature and being extremely exhausted. Owing to her continuing in this state and fearing septic poisoning the stump was dressed and, as in the other case, a large localised patch of recurrent gangrene presented. The wound was thoroughly cleansed with carbolic lotion and dressed as in the other case. She about this time took a turn for the better and the recurrent gangrene sloughed away, and after a considerable period the wound thoroughly healed by granulation. The patient slowly gained strength, and though her mental state has relapsed again she is in very fair bodily health and recently has been measured for an artificial limb.

My best thanks are due to Dr. Mott, who very kindly dissected the limb and cut sections of the vessels, and was able to determine in this last case the exact condition of the arteries and veins. He found that venous thrombosis was the chief cause, coupled with endarteritis of the larger arteries. Unfortunately we were unable to determine the cause microscopically in the first case, but Dr. Mott gave it as his opinion that stasis in the ressels was the most probable explanation.

Colney Hatch Asylum, New Southgate.

Str. Mungo's College Mredical Society, GlasGOW.-The following gentlemen were elected office-bearers for the year 1896-97 at the annual meeting held on Oct. 31st. Honorary President, Professor T. R. Marshall, D.Sc.; President, Mr. C. F. Spinks; Vice-Presidents, Dr. Duffus and Dr. Battersby; honorary secretary, Mr. F. P. Laslett; honorary treasurer, Mr. A. McCredie.

The Rahere Lodge of Freeliasons, No. 2546.An emergency meeting of this lodge was held at Frascati's Restaurant on Tuesday, Nov. 10th, Brother Clement Godsonacting for Brother Alfred Cooper, the W.M.--being in the chair. Brothers Cross, Willett, and Talérie were made Master Masons, and Brothers Keetley, Barron, and Nall were admitted to the second degree. Fifty members and visitors were present, of whom forty afterwards dined together. 